\title{
X-ray Powder Diffraction Studies of Mechanically Milled Cobalt
}

\author{
W.S. Yeo ${ }^{1, a}$, Z. NurAmirah ${ }^{2, b}$, H.S.C. Metselaar ${ }^{3, c}$ and T.H. Ong ${ }^{d}$ \\ ${ }^{1,2}$ Centre for Advanced Materials, University of Tenaga Nasional, Selangor, Malaysia. \\ ${ }^{3}$ Department of Mechanical Engineering, University of Malaya, Kuala Lumpur, Malaysia. \\ aprislera@hotmail.com, `amiraz13@gmail.com, chenkmetselaar@gmail.com, dprislera@yahoo.com.
}

\begin{abstract}
Keywords: Allotropic phase transformation, X-ray diffraction (XRD), high energy ball milling (BM), Cobalt, Rietveld method.
\end{abstract}

\begin{abstract}
The allotropic phase transformation of cobalt powder prepared by high-energy ball milling was investigated as a function of milling time. Measurement of crystallite size and microstrain in the powder systems milled for different times were conducted by X-ray diffractometry. The X-ray diffraction (XRD) peaks were analyzed using the Pearson VII profile function in conjunction with Rietveld method. X-ray diffraction line broadening revealed that allotropic transformation between face-centred-cubic phase (fcc) and hexagonal close-packed phase (hcp) in cobalt is grain size dependent and also on the accumulation of structure defects. The results showed that the phase formation of cobalt depends on the mill intensity that influences of both the grain size and the accumulation of structure defects. However, this theory alone is not adequate to explain the effects in this work. It was found that the total surface energy $(\Omega)$ theory satisfactorily explains the phase transformation behavior of cobalt. The smaller value of surface energy $(\Omega)$ of the fcc crystal than the hcp phase when size decreases may alter the qualitative aspects of the phase formation.
\end{abstract}

\section{Introduction}

High energy ball milling (BM) is an effective method for preparation of amorphous and nanocrystalline metal powders [1,2]. Several studies have revealed that nano-sized particles of some metals and alloys often showed anomalies in their behaviour with regard to phase transformation and phase stability, which are remarkably different from that in bulk materials [3]. It is well reported [4-7] that the allotropic phase transformation in cobalt is grain-size dependent. This theory alone is not adequate to explain the effects in this work since both phases found to occur at a range of grain size. Others reported that phase transformations occurring in cobalt when subjected to ball milling is found to be dependent on the milling intensity $[8,9]$. The result shows that with different milling intensities, a single fcc phase, single hcp phase or mixture of fcc and hcp phases could be formed. However, in this work, it was found that even at the same mill intensity the phase formation depends on milling time. The present work on the phase transformation of cobalt may be well explained according to Ram [10] theory of a further reduction of particle size below a critical value, which may modify its crystal structure and/or morphology in a manner that the latter is stable with a minimal value of its internal energy. At this scale, the fcc-Co will stabilize with a smaller value of total surface energy $(\Omega)$ over the hcp-Co structure. Therefore it is highlighted that the $\Omega$ plays a crucial role at this point. Based on these early literatures, it would appear that careful compositional and process control must be exercised in order to produce optimum results. It is strongly believed that the usefulness application of allotropic transformation to cobalt superalloy will be confined to specialized areas.

\section{Sample Preparation and Characterization}

In this work, ball milling of pure cobalt was carried out using a planetary ball mill (PM400, MA Type, Retsch) with a milling speed of $350 \mathrm{rpm}$ and at different milling times of up to 30 hours. The weight ratio of the ball-to-powder was fixed at 5:1. The cobalt particles with a mean size of $2 \mu \mathrm{m}$ were used as received in this study. 
The XRD was performed on a Philips X'Pert MPD X-ray diffractometer equipped with a $\mathrm{Cu}$ $\mathrm{K} \alpha$ radiation $(\lambda=0.154056 \mathrm{~nm})$. A peak profile standard material lanthanum hexaboride (SRM $660 \mathrm{a}-\mathrm{LaB}_{6}$ ) were used for instrumental correction. The profile fitting and the Rietveld analysis were performed using the program X'Pert HighScore Plus (HSP) which is develop by PANalytical Corporation, Netherland. The crystallite sizes, lattice strain and phase analysis were analyzed using Rietveld method. The Pearson VII function was selected for fitting the diffraction peak profile and a fourth order polynomial for background estimation. A direct comparison method is used to determine the volume fractions of various phases in a randomly orientated multiphase polycrystalline aggregate [11]:

$$
\frac{\mathrm{x}}{1-\mathrm{x}}=\mathrm{k} \cdot \frac{\mathrm{I}_{(200)}}{\mathrm{I}_{(101)}}
$$

in which $\mathrm{I}_{(200)}$ and $\mathrm{I}_{(101)}$ are the measured integrated intensities for (200) and (101) reflections, $\mathrm{k}$ is a constant depending on the radiation wavelength and $\mathrm{x}$ the volume fraction of the fcc phase.

The surface morphology of the milled powder was examined by Philips (XL 40) Scanning Electron Microscopy (SEM).

\section{Results and Discussion}

\subsection{X-ray Diffractometry Analysis of Milled Cobalt Samples.}

The XRD spectra of unmilled cobalt and milled cobalt are shown in Figure 1 with peaks at angle $2 \theta$ approximate of 47.4 and 51.6 belonging to (101) hcp and (200) fcc phase. The determination of the fcc and hcp phase are consistent with those mentioned in earlier work [12-13]. All initial samples consist of $26.05 \% \pm 2.91 \%$ of fcc cobalt phase. The fcc cobalt phase fraction in milled cobalt was $2.56,3.74,22.11$ and $19.94 \%$ for samples milled at 1, 5, 15 and 30 hours. The sample collected after 1 hour of milling shows no significant peaks-intensity at $2 \theta \approx 51^{\circ}$ which corresponds to the diffraction of (200) fcc cobalt. The absence of diffraction peaks associated with fcc cobalt indicates that the powder is transformed to the hcp state.

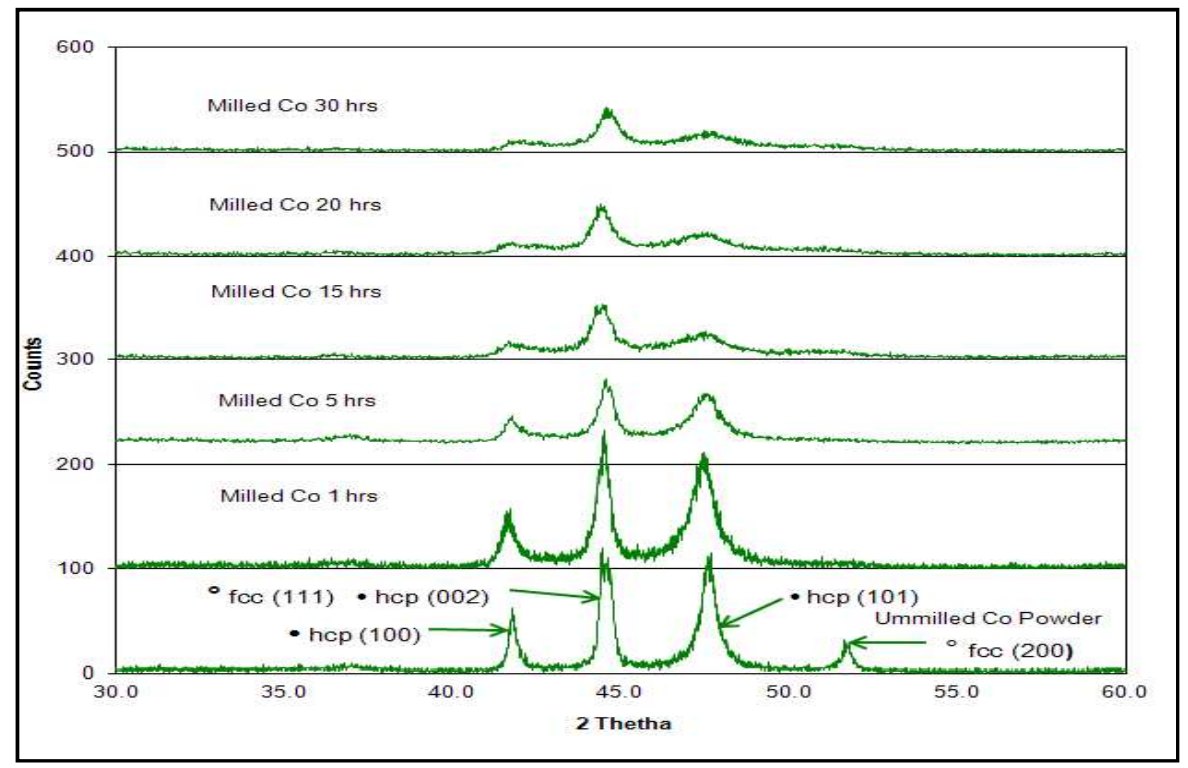

Figure 1 XRD Spectra of Unmilled Cobalt and Milled Cobalt Powders. 


\subsection{Profile Shape, Crystal Size and Lattice Strain Analysis of Milled Cobalt Samples.}

The variation of shape parameter, $\phi=2 \omega / \beta$, where $2 \omega=$ Bragg peak width (FWHM) at intensity equal to half the maximum intensity and $\beta=$ integral breadth [14] with milling time for the (101) line are shown in Figure 2. During initial milling (up to $\sim 1$ hours), the Cauchy profile increases rapidly and approaches towards the Cauchy limit $(\mathrm{CL}=0.479)$ which indicates the size reduction effect as an operative cause for overall line broadening.

For longer milling time ( $>15$ hours), the Gaussian profile increased with milling, approaching towards the Gaussian limit $(\mathrm{GL}=0.753)$, which signifies the strain effect for the overall profile broadening. At intermediate milling time (5-10 hours), both crystallite size and lattice strain affect the profile broadening. This is observed from the values of shape parameter which reveal their relative contribution varies with milling time. Therefore, for longer milling times their contribution is in a reverse manner.

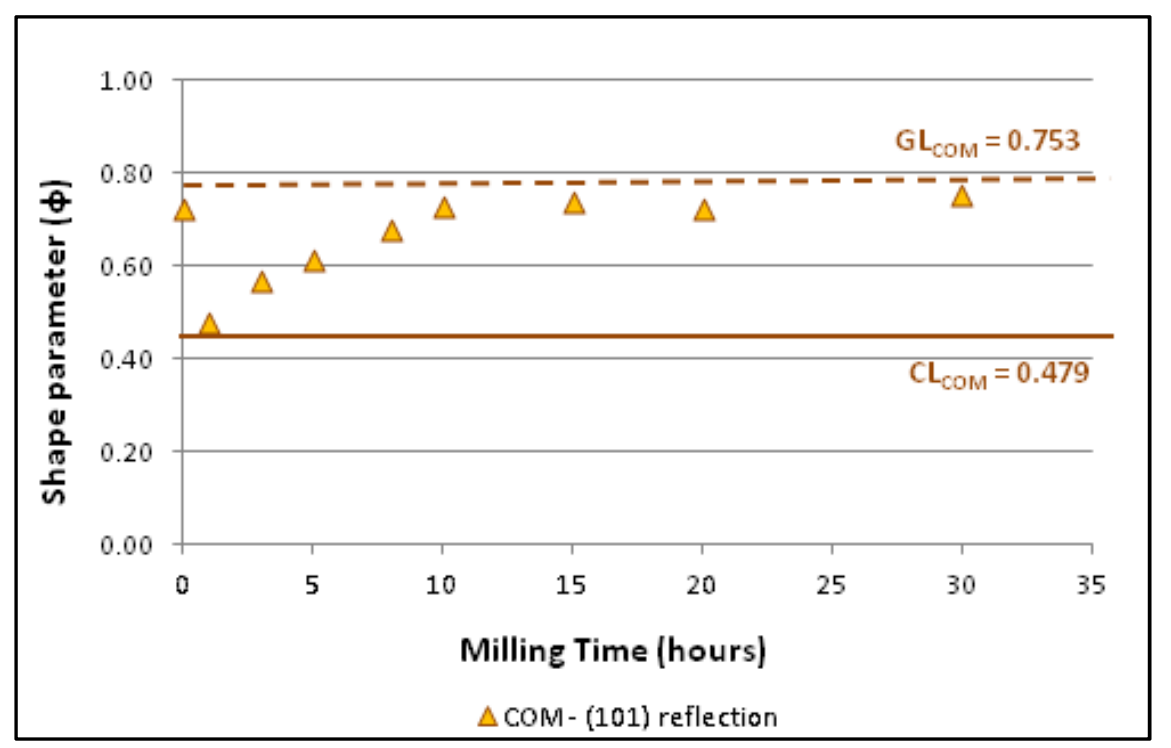

Figure 2 Variation of shape parameter with milling time along (101) line reflection.

These observations are confirmed by the trend of reduction of crystallite size and increases of lattice strain with milling time, which is shown in Figure 3. It was found that crystallite size and lattice strain have an inverse relationship, i.e. the largest values of crystallite size, the smallest values of lattice strain. In this case, approximately ten hours of milling time is required to attain the saturation crystallite size of $\sim 11-13 \mathrm{~nm}$ (by Rietveld). A further increase of the milling time from 10 to 30 hours does not caused any significant changes $( \pm 9 \%)$ of crystallite size.

It can be seen that from 1 to 10 hours of milling, the lattice strain increases rapidly in the cobalt powder. Similar to crystallite size behaviour, further milling (after 10 hours) does not induce significant changes $( \pm 6 \%)$ of lattice strain, but reached a saturation level of strain $(\sim 0.31 \%$ by Rietveld analysis). This is in agreement with the results of the surface energy (Table 1) of both structure which increases with milling time and, this is expected with the decreasing values of crystallite size with milling time. 


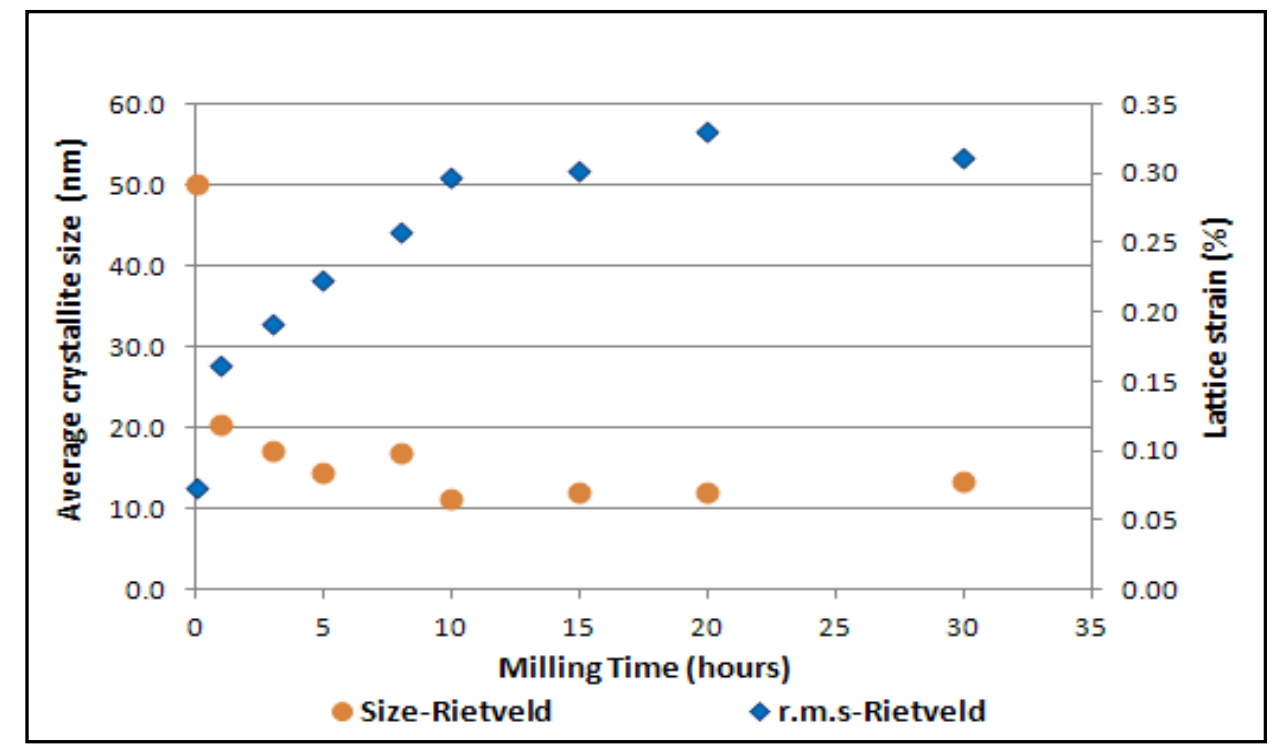

Figure 3 Average Crystallite Size and Lattice Strain vs. Milling Time by Rietveld Method.

Table 1 The Lattice Surface Area $(A)$ and Lattice Surface Energy $(\Omega)$ for hep and fcc crystal structures in milled cobalt powder.

\begin{tabular}{|c|c|c|c|c|}
\hline \multirow{2}{*}{$\begin{array}{c}\text { Milling Time } \\
\text { (hours) }\end{array}$} & \multicolumn{2}{|c|}{ hcp structure } & \multicolumn{2}{c|}{ fcc structure } \\
\cline { 2 - 5 } & $\begin{array}{c}\text { Surface area, } \\
A\left(10^{-2} \mathrm{~nm}^{2}\right)\end{array}$ & $\begin{array}{c}\text { Surface } \\
\text { energy, } \\
\Omega=A \sigma(\mathrm{J})\end{array}$ & $\begin{array}{c}\text { Surface area, } \\
A\left(10^{-2} \mathrm{~nm}^{2}\right)\end{array}$ & $\begin{array}{c}\text { Surface } \\
\text { energy, } \\
\Omega=A \sigma(\mathrm{J})\end{array}$ \\
\hline References value* & 93.88 & 259.66 & 75.39 & 208.52 \\
\hline 0 & 93.77 & 259.36 & 75.19 & 207.97 \\
\hline 1 & 94.26 & 260.71 & 75.15 & 207.86 \\
\hline 3 & 94.37 & 261.03 & 75.45 & 208.69 \\
\hline 5 & 94.54 & 261.49 & 75.64 & 209.21 \\
\hline 8 & 95.41 & 263.91 & 75.84 & 209.78 \\
\hline 10 & 95.59 & 264.40 & 75.99 & 210.20 \\
\hline 15 & 95.68 & 264.64 & 76.46 & 211.49 \\
\hline 20 & 95.45 & 264.03 & 76.34 & 211.14 \\
\hline 30 & 94.91 & 262.53 & 76.27 & 210.97 \\
\hline
\end{tabular}

* The value of $\Omega=A \sigma$ is calculated using the reported value of $\sigma=2.766 \mathrm{~J} / \mathrm{m}^{2}$ for polycrystalline cobalt structure [15].

It is observed that the increase of the surface energy was due to the increase of lattice parameter of the crystals when the size decreases, leading to the expansion of the cell. After 15 hours of milling, the lattice surface energy, $\Omega$, of hcp increased by a maximum value of $2 \%$ as compared with fcc phase of $1.7 \%$.It was found that the overall increase of total surface energy of single fcc phase is relatively smaller as compared to single hep phase. For both phases, a constant level of surface energy (i.e. $\Omega_{h c p}=\sim 264 \mathrm{~J}$ and $\Omega_{f c c}=\sim 211 \mathrm{~J}$ ) was reached after $\sim 10$ hours of milling, further milling up to 30 hours does not results in any significant $( \pm 0.3 \%)$ increase in lattice expansion and/nor surface energy.

\subsection{SEM Observations of Milled Nanocomposite Powders and Milled Cobalt Powders.}

The SEM micrographs of milled cobalt powders after 1 hour and 30 hours of milling times are shown in Figure 4(a) and (b) respectively. The larger agglomerate with exhibited a surface morphology clearly suggests that they were formed by coalescence of smaller particles through cold welding. The smooth appearance after longer milling time and the bigger size are indeed characteristic of ductile powders. The SEM examination of the MA-processed powder essentially 
does not substantiate the results inferred from the profile refinement. The SEM reveals that the powder agglomerations have lumps sizes ranging in micron meter and there are fine particles within these powder lumps.

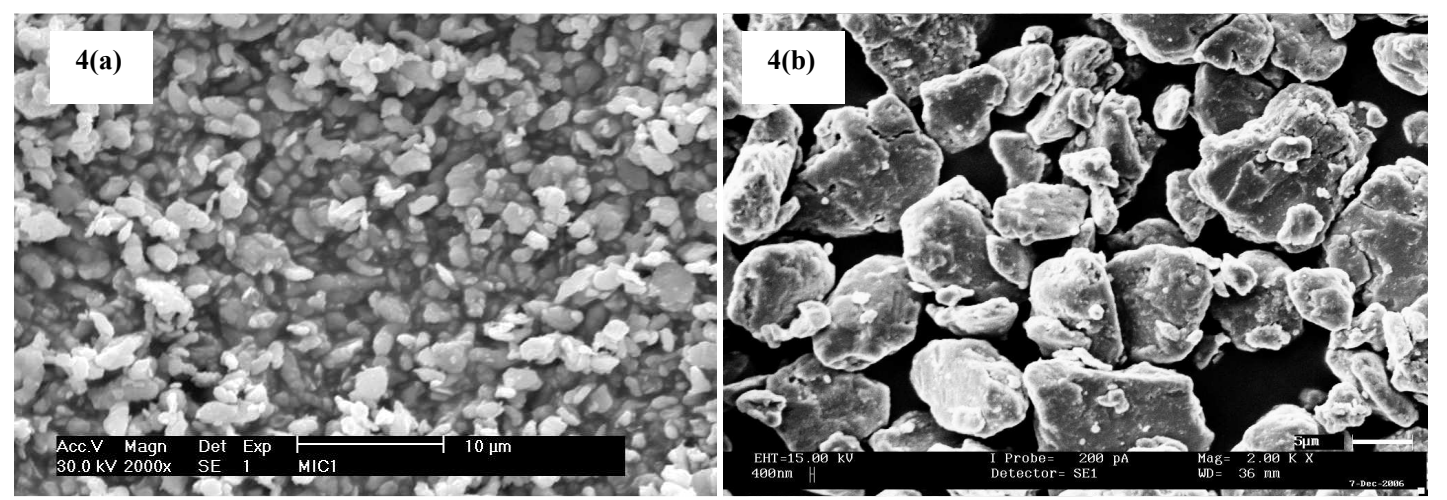

Figure 4(a) \& (b) SEM micrographs of cobalt powders obtained at 1 hour and 30 hours of milling, all at 2000x magnification.

\section{Conclusions}

In summary, cobalt is found to undergo hep-fcc allotropic phase transformation when subjected to mechanical milling. The allotropic phase transformation in cobalt depends on grain-size and accumulation of structure defects. Smaller crystallite size and increasing micro-strain lead to the formation of stable fcc structure. However, unless much effort was introduced to further reduce the crystallite size and increase the lattice strain, any higher percentage of fcc cobalt phase shall not be obtained. The total surface energy $(\Omega)$ theory is found satisfactorily explains the allotropic phase formation behaviour of cobalt. The fcc cobalt phase has a smaller value of $\Omega$ than the hep phase and becomes predominant as the particle size decreases.

Acknowledgments: The award of $\mathrm{PhD}$ scholarship for W.S. Yeo from University Tenaga Nasional is acknowledged.

\section{References}

[1] M. Jachimowicz, V. I. Fadeeva and H. Matyja: Nanostructured Materials Vol. 12 (1999), p.159

[2] Z. Lidong, Z. Jochen and L. Erich: Surface and Coatings Technology Vol. 168 (2003), p.179

[3] H. Gleiter: Nanostructured Materials Vol. 6 (1995), p.3

[4] O. Kitakami, H. Sato and Y. Shimada: Physical Review Vol. 56 (1997), p.13849

[5] A.R Troiano and J.L. Tokich: American Institute of Mining, Metallurgical, and Petroleum Engineers Vol.175 (1948), p.728

[6] E.A. Owen and D. Madoc Jones: Proceedings of Phys. Soc. B 67 (1954), p.456

[7] O.S. Edwards and H. Lipson: J. Inst. Metals Vol. 96 (1943), p.177

[8] J.Y. Huang, Y.K. Wu, H.Q. Ye and K. Lu: Nanostructure Materials Vol. 6 (1995), p.723

[9] J.Y. Huang, Y.K. Wu and H.Q. Ye: Acta Materialia Vol. 44 (1996), p.1201

[10] S. Ram, D. Ghosh and S.K. Roy: Journal of Materials Science Vol. 36 (2001) p.3745

[11] Amir A. K, Dissertation, University of Toronto (2001).

[12] W.S. Yeo and H.S.C. Metselaar: Synthesis and Reactivity in Inorganic, Metal-Organic, and Nano-Metal Chemistry Vol.38 (2008), p.341

[13] W.S. Yeo and H.S.C. Metselaar: Proceedings of $6^{\text {th }}$ Interrnational Conference on Materials Processing, Properties and Performance (MP3) (2007).

[14] Th.H. De Keijser, J.I. Langford, E.J. Mittemeijer and A.B.P. Vogels: Journal of Applied Crystallographic Vol.15 (1982), p.308

[15] W. Betteridge: Progress in Materials Science Vol.24 (1979), p.51. 